UTHEP-497

UTCCS-P-9

\title{
Charmed meson spectra and decay constants with one-loop $O(a)$ improved relativistic heavy quark action *
}

\author{
CP-PACS Collaboration : Y. Kayaba, S. Aoki, M. Fukugita, ${ }^{\mathrm{b}}$ K-I. Ishikawac ${ }^{\mathrm{a}}$ Y. Iwasaki, K. Kanaya, ${ }^{\mathrm{a}}$, \\ T. Kaneko, Y. Kuramashi, ${ }^{\text {, e }}$ M. Okawa, A. Ukawa, ${ }^{a}$, T. Yoshié,, e \\ ${ }^{\text {a }}$ Grad. School of Pure and Applied Sciences, University of Tsukuba, Tsukuba, Ibaraki 305-8571, Japan \\ b Institute for Cosmic Ray Research, University of Tokyo, Kashiwa, Chiba 277-8582, Japan \\ c Department of Physics, Hiroshima University, Higashi-Hiroshima, Hiroshima 739-8526, Japan \\ d High Energy Accelerator Research Organization (KEK), Tsukuba, Ibaraki 305-0801, Japan \\ e Center for Computational Sciences, University of Tsukuba, Tsukuba, Ibaraki 305-8577, Japan
}

\begin{abstract}
We calculate charmed meson spectra and decay constants in lattice QCD employing one-loop $O(a)$ improved heavy quark action and axial-vector currents. In quenched simulations at $a \sim 0.1 \mathrm{fm}$ with the plaquette gauge action as well as a renormalization-group improved one, it is shown that the deviation from the continuum dispersion relation and the violation of space-time symmetry for the pseudoscalar meson decay constants are substantially reduced, once the $O(a)$ improvement is applied. Preliminary results with two flavors of dynamical quarks are also presented.
\end{abstract}

\section{Introduction}

With current computational resources it is difficult to achieve a precise determination of weak matrix elements associated with $B$ and $D$ mesons because of large $m_{Q} a$ corrections for $b$ - and $c$-quarks. Recently a relativistic heavy quark(RHQ) action was proposed to control these $m_{Q} a$ corrections by applying the on-shell $O(a)$ improvement program to massive quarks [1, and the mass-dependent improvement coefficients have been perturbatively determined up to oneloop level[2]. The improvement of the heavyheavy and heavy-light axial vector currents are also carried out up to one-loop level in a mass dependent way $[3$. In this report we perform a numerical study of the RHQ action in quenched QCD by investigating the effective speed of light $c_{\text {eff }}$ from the meson energy and the space-time asymmetry $R$ for the pseudoscalar decay constant. The deviation from $c_{\text {eff }}=1$ and $R=1$ measures typical systematic errors due to finite $m_{Q} a$ corrections left in the formulation, which should be incorporated in the results of charmed

*Talk presented by Y. Kayaba meson spectra and decay constants. Preliminary results with two flavors of dynamical quarks are also presented.

\section{Simulation details}

We employ the clover(CL) action with nonperturbative $c_{\mathrm{SW}}^{\mathrm{NP}}$ 45] for light quarks and the RHQ action for heavy quarks combined with the plaquette and the Iwasaki gauge actions. It is worth mentioning that we replace the massless part of the one-loop improvement coefficients $c_{B}$ and $c_{E}$ in the RHQ action with that of the nonperturbative one as

$c_{B / E}=c_{B / E}^{\mathrm{PT}}\left(m_{Q} a\right)-c_{B / E}^{\mathrm{PT}}(0)+c_{\mathrm{SW}}^{\mathrm{NP}}$,

where the superscript PT represents "perturbative" value. With this replacement $O(a)$ errors are completely removed at $m_{Q}=0$. We calculate charmed meson spectra using 300 configurations on a $24^{3} \times 48$ lattice at $a \sim 0.1 \mathrm{fm}$ in quenched QCD. Three values of the lihgt quark mass corresponding to $M_{P S} / M_{V} \sim 0.56-0.77$ and four values of the heavy quark mass around the charm quark mass are adopted. For comparison we per- 


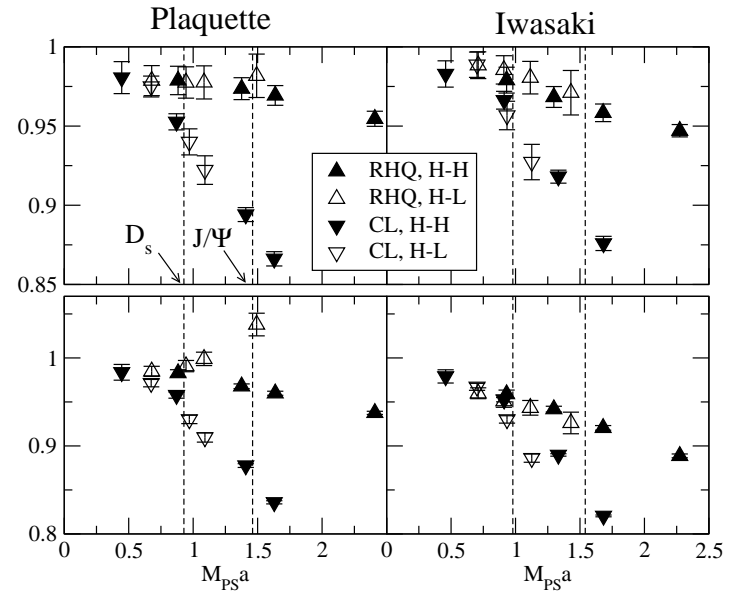

Figure 1. Effective speed of light for the PS meson(top) and space-time asymmetry for the PS meson decay constant(bottom) as a function of the meson mass in quenched QCD.

form parallel calculations with the CL action for both heavy and light quarks at the same gauge coupling and lattice size.

\section{Numerical study of the RHQ action}

Let us examine effectiveness of the $O(a)$ improvement for the RHQ action in a nonperturbative way. We extract the effective speed of light $c_{\text {eff }}$ by fitting the pesudoscalar(PS) meson energy $a E$ as a function of spatial momentum $a p_{s}$ :

$(a E)^{2}=\left(a M_{\text {pole }}\right)^{2}+c_{\text {eff }}^{2}\left(a p_{s}\right)^{2}$,

where $M_{\text {pole }}$ denotes the pole mass. $c_{\text {eff }}$ is expected to become unity in the continuum limit. Numerical results are plotted in Fig. 1(top) as a function of the meson mass for heavy-heavy $(\mathrm{H}-\mathrm{H})$ and heavy-light(H-L) systems. Around charmed meson mass the deviation from $c_{\text {eff }}=1$ is equal to or larger than about $10 \%$ for the CL heavy quark action, while less than $5 \%$ for the RHQ action.

We also measure the space-time asymmetry in terms of the PS meson decay matrix elements:

$R \equiv i \frac{\left\langle 0\left|A_{k}^{R}\right| P S\right\rangle}{\left\langle 0\left|A_{4}^{R}\right| P S\right\rangle} \cdot \frac{E}{\left|p_{s}\right|}$,

which should become unity in the continuum limit. Results are plotted in Fig 1 (bottom) as

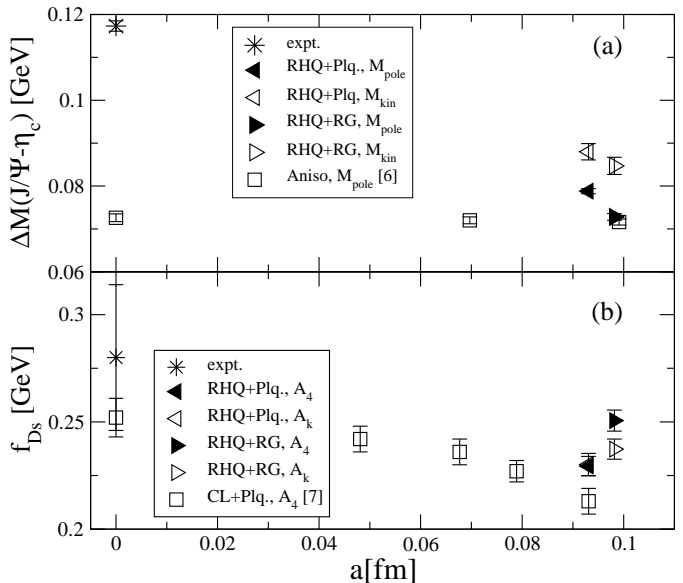

Figure 2. Charmonium S-state hyperfine splitting(top) and $D_{s}$ meson decay constant(bottom) in quenched QCD as a function of $a$ set by Sommer scale $r_{0}$.

a function of the meson mass. The asymmetry reaches $10 \sim 20 \%$ around the charmed meson mass for the CL action, while it is less than $8 \%$ for the RHQ action. These observations allow us to conclude that the improved action works as designed in reducing the $m_{Q} a$ corrections.

Figure 2(a) shows the charmonium S-state hyperfine splitting as a function of lattice spacing, where we practice two analysis: one is based on the pole mass of the charmed meson and another on the kinetic mass:

$a M_{\text {kin }}=a M_{\text {pole }} / c_{\text {eff }}^{2}$.

We find $15 \%$ discrepancy, which should be included as a systematic error. Compared to results with anisotropic lattice QCD [], our results are slightly larger at $a \sim 0.1 \mathrm{fm}$. Both are substantially lower than the experimental value.

In Fig. 2(b) we plot the $D_{s}$ meson decay constant which are obtained from the time and spatial components of the axial vector current. The difference between two definitions is less than $3 \%$ for both gauge actions. For comparison we also plot the results obtained by the CL heavy quark action with the plaquette gauge action[7]. Our results at $a \sim 0.1 \mathrm{fm}$ are much closer to the value of the CL heavy quark action in the continuum limit than at a similar lattice spacing. This would 


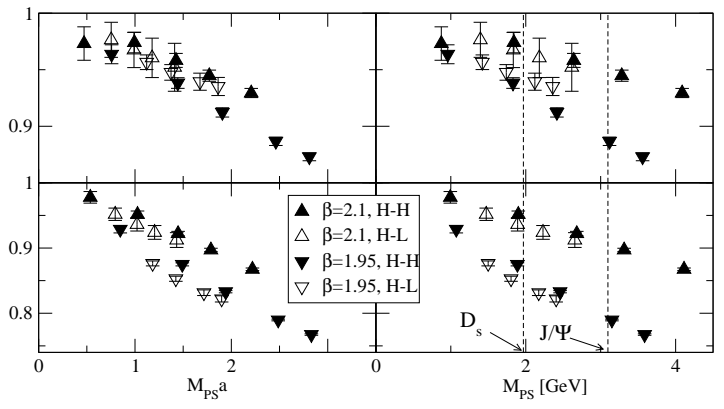

Figure 3. Effective speed of light for the PS meson(top) and space-time asymmetry for the PS meson decay constant(bottom) as a function of the meson mass in lattice unit(left) and physical unit(right) for $N_{f}=2$ QCD.

suggest that the RHQ action indeed improves the scaling behavior of the decay constant.

\section{Preliminary results of $N_{f}=2 \mathrm{QCD}$}

Encouraging results in quenched QCD urge us to apply the calculation to the two-flavor QCD gauge configurations [8]. We employ the meanfield improved CL light quark action and the RHQ action for the valence quraks. Up to now two set of lattices have been analyzed: 400/200 configurations for $16^{3} \times 32 / 24^{3} \times 48$ at $\beta=$ $1.95 / 2.1$ with four sea quark masses. Lattice spacings are roughly $0.15 \mathrm{fm}$ and $0.11 \mathrm{fm}$, respectively. We make a similar choice for the set of valence heavy and light quark masses as in the quenched case.

In Fig. 3 we show the effective speed of light and the space-time asymmetry for the PS meson decay constant. As a decreases, the deviation from $c_{\text {eff }}=1$ and $R=1$ is reduced at fixed $m_{Q} a$ and $m_{Q}$. These behavior are consistent with the expected leading scaling violation of the RHS action given by $O\left(f_{2}\left(m_{Q} a\right)\left(a \Lambda_{\mathrm{QCD}}\right)^{2}\right)$ or $O\left(f_{1}\left(m_{Q} a\right) g^{4} a \Lambda_{\mathrm{QCD}}\right)$, where $f_{1}$ and $f_{2}$ are some unkown functions. A little surprising is that, as seen from the left panels in Fig. [3 $f_{1,2}$ look almost linear in terms of $m_{Q} a$ even beyond $m_{Q} a=1$.

Figure 4 illustrates the $a$ dependence of the $D_{s}$ meson decay constant. While the scaling behavior from $A_{4}$ with the RHQ action is only a little

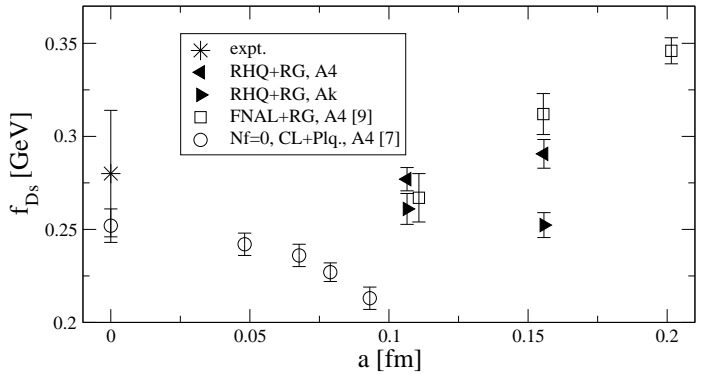

Figure 4. $D_{s}$ meson decay constant for $N_{f}=2$ QCD as a function of $a$. The scale is set by $r_{0}$ for our results and $m_{\rho}$ for FNAL method.

milder over the previous result obtained by the FNAL interpretation with the CL heavy quark action [9], the one from $A_{k}$ is much improved. This result suggests that we will be able to make a reliable continuum extrapolation, employing a simultaneous fit for $f_{D_{s}}$ obtained from $A_{4}$ and $A_{k}$, once we have data on CP-PACS configurations at $\beta=2.2$ or 1.8 .

This work is supported in part by the Grantsin-Aid for Scientific Research from the Ministry of Education, Culture, Sports, Science and Technology. (Nos. 13135204, 13640260, 14046202, $14740173, \quad 15204015, \quad 15540251, \quad 15540279$, 16028201, 16540228, 16740147, )

\section{REFERENCES}

1. S. Aoki, Y. Kuramashi and S. Tominaga, Prog. Theor. Phys. 109 (2003) 383.

2. S. Aoki, Y. Kayaba and Y. Kuramashi, Nucl. Phys. B 697 (2004) 271.

3. S. Aoki, Y. Kayaba and Y. Kuramashi, Nucl. Phys. B 689 (2004) 127.

4. M. Lüscher, S. Sint, R. Sommer, P. Weisz and U. Wolf, Nucl. Phys. B 491 (1997) 323.

5. CP-PACS Collaboration: S. Aoki et al., in preparation.

6. CP-PACS Collaboration: M .Okamoto et al., Phys. Rev. D65 (2002) 094508.

7. ALPHA Collaboration: A. Juttner et al., Phys. Lett. B560 (2003) 59

8. CP-PACS Collaboration: A. Ali Khan et al., Phys. Rev. D65 (2002) 054505

9. CP-PACS Collaboration: A. Ali Khan et al., Phys. Rev. D64 (2001) 034505 